Chapman University

Chapman University Digital Commons

7-2021

\title{
Handwriting Correction System using Wearable Sleeve with Optimal Tactor Configuration
}

Dhanya Nair

Grant Stankaitis

Sean Duback

Robert Geoffrion

Justin B. Jackson

Follow this and additional works at: https://digitalcommons.chapman.edu/engineering_articles

Part of the Biomechanical Engineering Commons, Disability and Equity in Education Commons, Other Engineering Commons, and the Special Education and Teaching Commons 


\section{Handwriting Correction System using Wearable Sleeve with Optimal Tactor Configuration}

\section{Comments}

This is a pre-copy-editing, author-produced PDF of an article accepted for publication in the 2021 18th International Conference on Ubiquitous Robots (UR). The definitive publisher-authenticated version is available online at https://doi.org/10.1109/UR52253.2021.9494651.

\section{Copyright}

(c) 2021 IEEE. Personal use of this material is permitted. Permission from IEEE must be obtained for all other uses, in any current or future media, including reprinting/republishing this material for advertising or promotional purposes, creating new collective works, for resale or redistribution to servers or lists, or reuse of any copyrighted component of this work in other works. 


\title{
Handwriting Correction System using Wearable Sleeve with Optimal Tactor Configuration
}

\author{
Dhanya Nair, Grant Stankaitis, Sean Duback, Robert Geoffrion, and Justin B. Jackson, Member, IEEE
}

\begin{abstract}
Handwriting remains an elusive skill with practice worksheets being the common method of learning. Since these worksheets provide only visual feedback and no quantitative feedback, it can often be a challenge to improve. For children with learning disabilities, learning handwriting skills is one of the most difficult tasks. We propose a handwriting training system that uses off-the-shelf webcam, a pen tracking software and a haptic sleeve which provides active feedback to the user based on their deviation from the original pattern. The sleeve has 4 individual motors that vibrate at different intensities based on the direction (right, left, up or down) and severity of the deviation $(<1 \mathrm{~cm}, 1 \mathrm{~cm}-3 \mathrm{~cm},>3 \mathrm{~cm})$. Different motor placements around the forearm are evaluated for vibro-tactile feedback accuracy and time response, and a novel spaced-ring configuration is proposed. This paper provides details on the system architecture and sleeve characterization, and the results show promise in utilizing the system for self-correction and visual-motor skills development. The results from sleeve characterization suggest the applicability of the spaced-ring configuration (perceived feedback accuracy $>\mathbf{9 8 \%}$, time response $<1$ s) in other vibrotactile hand guidance systems, in addition to handwriting correction. Recommendations on tactor placements around the forearm are provided.
\end{abstract}

\section{INTRODUCTION}

Handwriting skills are usually developed during the primary years of a child's schooling. However, it is a major struggle for children lacking motor skills or facing physical/cognitive challenges [1]. Assistive handwriting training devices can not only help children with learning disabilities but could play a major role in rehabilitation of individuals affected by loss of fine motor skills due to neurological conditions [2]. Bara et al. [3] found that systems capable of providing haptic feedback in addition to visual representation were more useful for handwriting training than those providing visual alone. Multimodal sensory feedback is highly beneficial for developing strong visual-motor skills (a prerequisite to handwriting training) [3, 4] and for character retention [5]. Several systems have been developed to provide haptic guided handwriting training for children and stroke patients [2, 5-7]. Studies evaluating the different methods of providing haptic guidance (partially guided and fully guided) for handwriting training have demonstrated that combining these methods over the training period yields better results than using either of them alone [8], and each method is better suited for a different group of alphabets (classified based on its level of complexity) [9]. Due its easy access, Phantom Omni has almost exclusively been the development platform for these systems [1-9].

D. Nair is with the Fowler School of Engineering, Chapman University, Orange, CA 92866, USA. (phone: 806-224-5882; e-mail: n.danya@gmail.com,dnair@chapman.edu).

G. Stankaitis was with Chapman University. He is now with Department of Electrical Engineering, University of Hawaii at Manoa, HI 96822, USA.
Given the published results on the applicability of assistive haptic devices in handwriting training [1-9], we propose a lowcost wearable device (haptic sleeve) for assisted handwriting training. To the best of authors' knowledge, most wearable handwriting training systems are focused only on handwriting recognition and not on providing real-time feedback [10-12]. The commercially available devices for assistive handwriting rely on additional weight provided to the palm or wrist for increased spatial awareness, or in changing the grip [13, 14]. Morikawa et al. and Narita et al. $[15,16]$ have demonstrated the ability of utilizing a wrist-worn system (providing pressure feedback) in calligraphy training. Their systems utilized Leapmotion sensor whereas our design works with any off-theshelf webcam. While prior studies utilize Phantom Omni to help children with handwriting patterns, we hypothesize that a wireless glove/sleeve system will be advantageous due to 1) receiving gentle vibrations on the wrist might 'feel' more close to the natural learning environment with an educator holding the hand and providing corrections, and 2) sleeves provide complete freedom to the individual's hand movement (untethered environment) and have more likelihood of being adopted as a long term training tool.

This paper details the architecture of the prototype and the design of the sleeve. Section II provides the software and hardware architecture; section III presents the results from testing and characterization of the sleeve, section IV discusses the results and gives insights for optimal tactor placements on a sleeve, and section $\mathrm{V}$ concludes with a summary and work remaining.

\section{System ARCHITECTURE}

\section{A. System Overview}

The assisted handwriting training system described in this paper consists of a software (utilizing webcam) that tracks the user's pen movement as they attempt to trace a pattern. A haptic sleeve is worn by the user and has 4 motors that provide immediate vibratory feedback to the user if they deviate from the original pattern. The vibrations increase as they deviate further from the correct path and decrease as they move closer.

The user writes on a $8.5 \times 11$ inch transparent glass plate placed on a stand with the webcam beneath it. The data from the webcam is sent to our learn-to-write software where the pen's deviation (distance and direction) from the correct path

S. Duback, R. Geoffrion, and J. Jackson are with Department of Electrica \& Computer Engineering, Weber State University, UT 84408, USA 
is computed. The distance and direction data are sent to the sleeve via Bluetooth Low Energy (BLE) (Fig. 1).

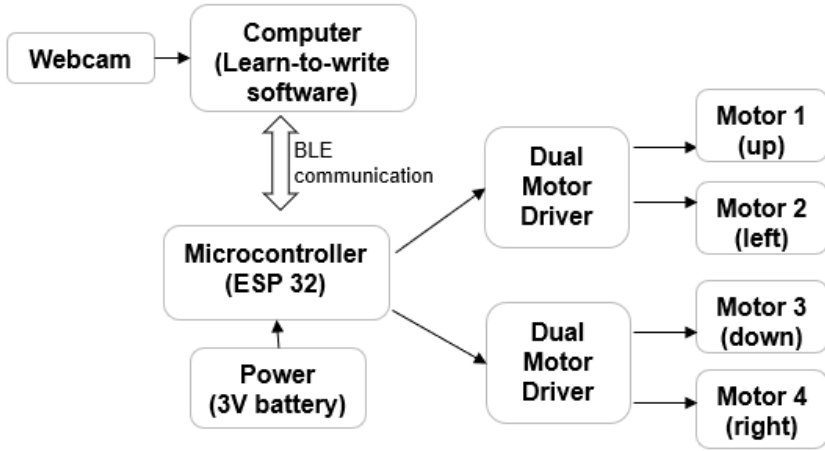

Figure 1. Overall System Architecture

\section{B. Learn-to-write Software}

An image containing the pattern to be traced is input to the system. Although the software can operate on any image with contours, an image of the letter $\ell$ printed in Microsoft paint using Script font is used for demonstration purposes. The given image is converted to a black and white .png image (to preserve the color values of every pixel) by the software. The software is designed to track an orange marker (orange circular object). It continuously monitors the webcam input for the presence of an orange circle (Fig. 2). Once detected, it stores the location ( $\mathrm{x}, \mathrm{y}$ coordinates) of the center of the circle as the starting point (Fig. 3).

Since it was computationally inefficient to compare the user input to every contour pixel in the original pattern, the black and white contour image is split into separate parts called "decision windows". The decision windows were created by starting with a $3 \times 3$ pixel area containing contour pixels and growing the size by including neighboring pixels if they contained contour pixels but without crossovers. The optimal number of decision windows and their sizing was determined empirically (Fig 4.a).

At the start, only the decision window nearest to the marker is made active (only the contour within the active frames is utilized by the software for computation). The software continuously tracks the orange marker and computes the Euclidean distance (in pixels) from its center to all the contour pixels (black pixels) in the active window(s) (Fig. 4.b). Once the hand moves and the distance of the marker to the current window increases, there onwards two decision windows nearest to the marker are always kept active. The active window with the lower distance from the marker is designated as the current window and the other one as the next window. As the marker moves away from the current window and closer to the neighboring window, the neighboring window becomes the new current window, and a new next window is activated (Fig. 5). The software displays the pattern traced by the user directly on top of the original pattern so that the user has multiple (visual and tactile) feedbacks for self-correction (Fig. 4.c).

The Euclidean distance of the marker from the original pattern is computed and compared to the threshold for correction. This distance is classified into one of 3 segments (close is $<1 \mathrm{~cm}$, further is $1 \mathrm{~cm}-3 \mathrm{~cm}$, far as $>3 \mathrm{~cm}$ ) determined empirically. By comparing $\Delta \mathrm{x}$ ( $\mathrm{x}$-coordinates of marker minus contour) and $\Delta y$ values, the direction of deviation is determined to be left, right, up, or down encoded into integer values (Fig. 2).

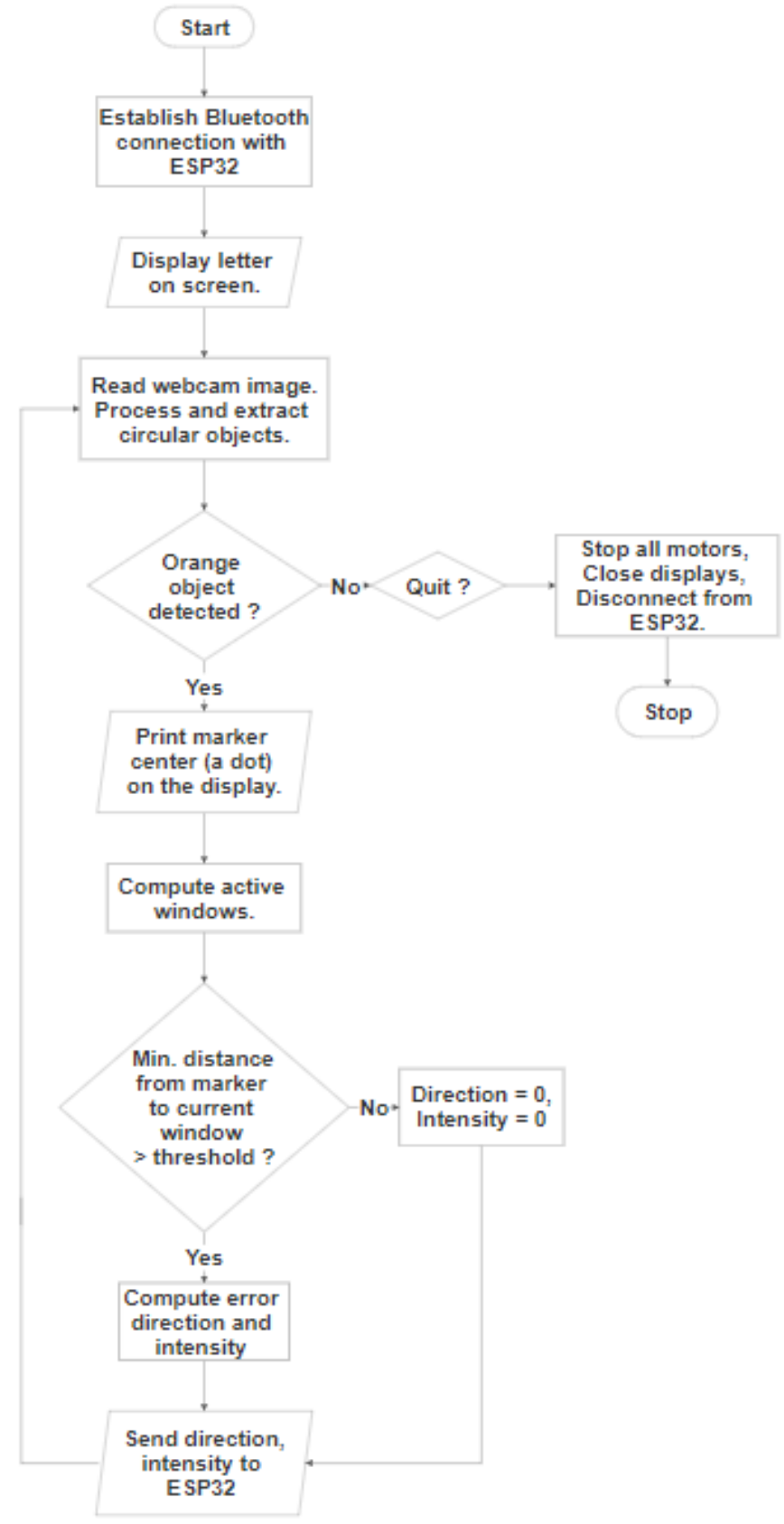

Figure 2. Learn-to-write software flowchart

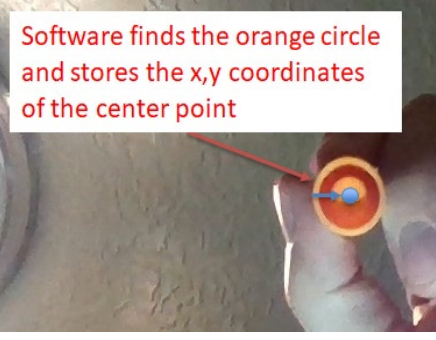

Figure 3. Software tracking orange circle 


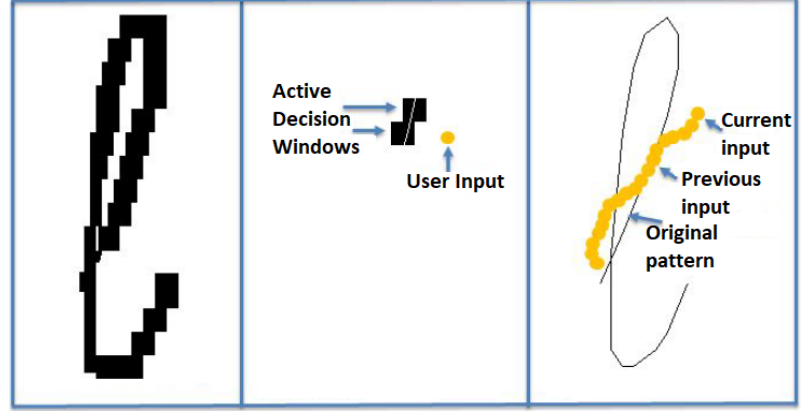

Figure 4 (a) All 35 decision windows for letter $l$ (b) The two active windows (c) The visual output to the user.

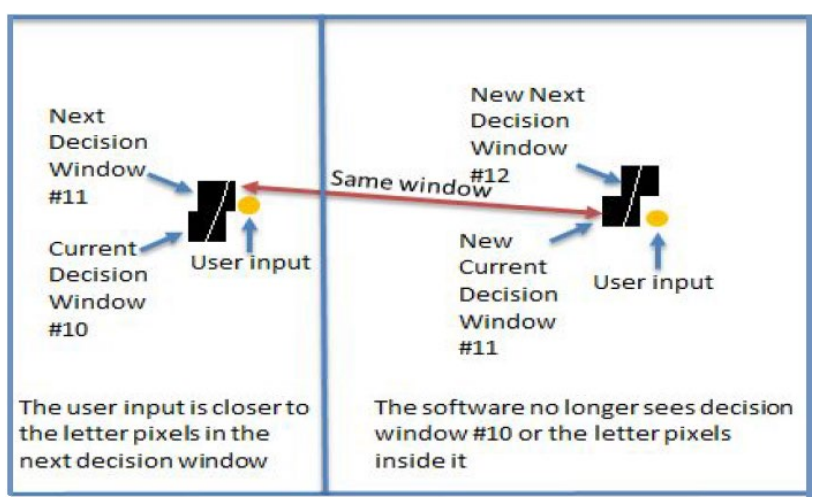

Figure 5. Determining new current window and next window.

\section{Hardware}

The sleeve has pockets to hold a small PCB with microcontroller and 2 motor drivers, 2 AA batteries (or $3.3 \mathrm{~V}$ lithium-ion battery), and 4 vibrating mini disk motors (ADA 1201) (Fig. 6). The ESP32 microcontroller is designed for wearable electronics with low-cost, low-power, and built-in Wi-Fi and BLE capability. The dual-motor drivers (TB6612FNG) are each capable of controlling 2 motors individually while providing the necessary power to each. Different motor configurations were considered, and the spaced-ring configuration described below provided best results during user testing. In the spaced ring configuration, the up motor was placed near the wrist on the dorsal side, the down motor was placed on the volar side away from the wrist (near the proximal side of the sleeve), the left and right motors were placed midway between the up and down motors on the respective sides of the forearm.

Whenever there is a change in the error direction or distance, the learn-to-write software sends 2 integer values (direction, distance) to the ESP32 via BLE. Based on the direction received, one of the 4 motors is activated at one of 3 intensity levels. The motor's intensity levels are controlled using pulse width modulation (PWM) with a $250 \mathrm{~Hz}$ signal varied among 3 different duty cycles $(33 \%, 66 \%, 100 \%)$ corresponding to the distance (close, further, far).
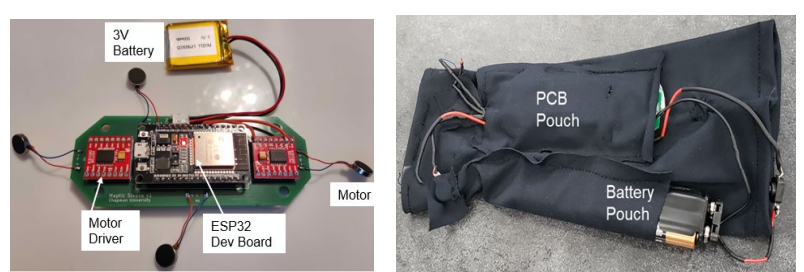

Figure 6 (a) Printed circuit board (PCB) design (b) Sleeve with embedded PCB, battery and motors.

\section{Sleeve Design}

For self - correcting the hand movement, it is important for the user to accurately distinguish the location of motor activation and be able to respond quickly. To determine the best motor positions around the wrist/forearm that provide maximum discrimination between the different directions, four different arrangements were considered: (i) Ring arrangement around the wrist, (ii) Top-arm arrangement, (iii) Bottom-arm arrangement, and (iv) Spaced-ring arrangement around the forearm.

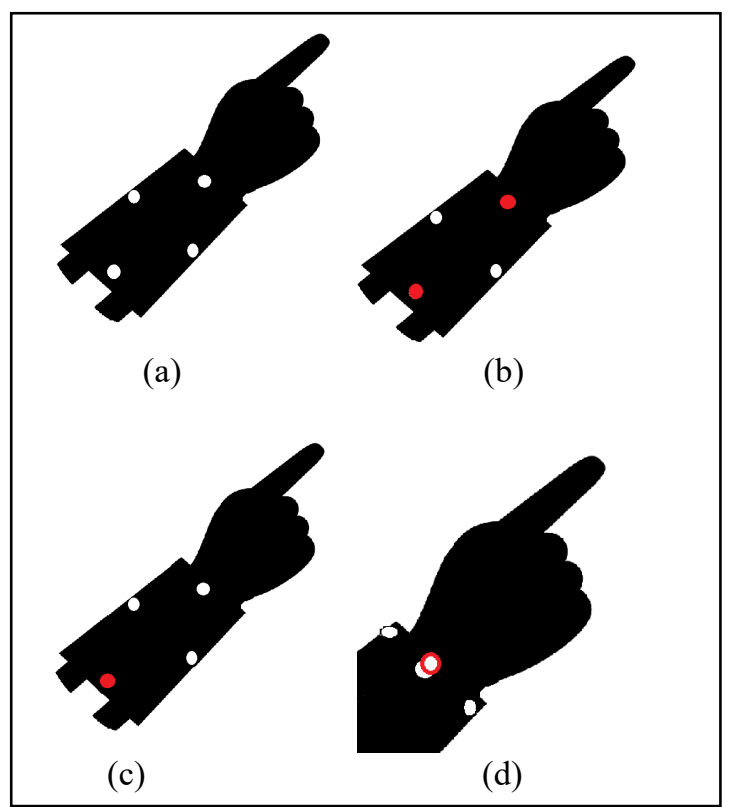

Figure 7. Placement positions of the 4 motors on the sleeve in different configurations: (a) Top arm configuration (b) Bottom arm configuration (c) Spaced ring configuration (d) Wrist ring configuration. White dots correspond to motors placed on the dorsal side, or the radial/ulnar sides. Red dot indicates the motor is placed on the volar side.

In the wrist-ring arrangement (Fig. 7.d), the motors were placed around the 4 quadrants of the wrist (dorsal, ventral, radial, ulnar sides) as presented in literature [17]. Since the two-point discrimination on the forearm is between $25 \mathrm{~mm}$ $38 \mathrm{~mm}[18,19]$, the remaining configurations spaced the motors by $50 \mathrm{~mm}$ or more.

In the top-arm arrangement (Fig. 7.a), the up and down motors were both placed on the dorsal side of the forearm, with one near the wrist and the other $100 \mathrm{~mm}$ away on the proximal region. The left and right motors were placed $50 \mathrm{~mm}$ away from the wrist (i.e. midway between the up and down motors) on the radial and ulnar side respectively. The left and right motors were placed on the same location for the bottom arm and spaced ring arrangement. In the bottom-arm arrangement (Fig. 7.b), both the up and down motors were placed on the volar side of forearm (100mm apart) with none on the dorsal side. In the spaced-ring arrangement (Fig. 7.c), the up motor was placed near the wrist on the dorsal side and the down motor was placed on the volar side, $100 \mathrm{~mm}$ away from the wrist motor, near the proximal side of the sleeve/forearm. 


\section{TEST METHODS}

The different configurations of the sleeve and the overall system were tested on 3 healthy adults ( 2 female, 1 male) with an average age of 35 years. All participants were right-handed and wore the sleeve on their right hand.

The participants were asked to wear the sleeve (with the motors embedded in the desired configuration) and the motors were activated randomly, one at a time. The participants were asked to identify the activated motor and press the corresponding up/down/left/right arrow key on a keyboard as soon as they identified the direction. The intensity of vibration was kept constant at $50 \%$ duty cycle.

Once activated, the motors remained active till the user provided a response. Hence, the same motor was not activated twice in succession but otherwise the order of motor activation was randomized. Each participant performed 2 iterations of the accuracy test on each of the 4 configurations. During each iteration, 100 trials were conducted with each motor/direction being activated 25 times. The order of the configuration/sleeve tested was varied between the different participants and between the 2 iterations for the same participant. The participants were given 10-minute break between testing each configuration.

For each of the sleeve configurations, a confusion matrix was created to analyze the accuracy and any directional bias. The elapsed time between the motor actuation and the user response was also evaluated for all trials. Results from this response speed testing and the accuracy testing were used to determine the optimal sleeve configuration.

\section{PRELIMINARY RESUltS}

To evaluate the optimal configuration for placing the tactors around the wrist/forearm, the analysis criteria were 1) determine how accurately the participants were able to distinguish the activated motor/direction for different configurations and 2) determine if there was a difference in user response speed for the different configurations. This section presents the results from these analyses.

\section{A. Motor Configuration Accuracy}

For every sleeve configuration, each participant provided 50 responses ( 25 times $\times 2$ iteration) to the activation of each of the 4 motors. Their cumulative response is shown in Figure 8 . For all the 4 configurations, the participants were able to distinguish the activated motor/direction with an accuracy of $90 \%$ or above.

However, there was a higher tendency of misclassification of the down direction in the case of Top-arm configuration (Fig. 8.a.). A similar bias was observed in the Bottom-arm configuration wherein the up motor was misclassified more often (Fig. 8.b). The wrist - ring configuration had the maximum confusion out of the 4 configurations (Fig. 8.d). The spaced-ring configuration provided highest accuracy (98\%) with no significant bias in either of the 4 directions (Fig. 8.c, Fig. 9).

\begin{tabular}{|c|c|c|c|}
\hline 144 & 0 & 3 & 3 \\
\hline 0 & 148 & 2 & 0 \\
\hline 0 & 10 & 135 & 5 \\
\hline 0 & 0 & 3 & 147 \\
\hline
\end{tabular}

(a)

\begin{tabular}{|c|c|c|c|}
\hline 148 & 2 & 0 & 0 \\
\hline 0 & 148 & 1 & 1 \\
\hline 0 & 1 & 148 & 0 \\
\hline 0 & 0 & 4 & 146 \\
\hline
\end{tabular}

(c)

\begin{tabular}{|c|c|c|c|}
\hline 137 & 6 & 6 & 1 \\
\hline 6 & 143 & 0 & 1 \\
\hline 0 & 0 & 148 & 2 \\
\hline 0 & 0 & 2 & 148 \\
\hline
\end{tabular}

(b)

\begin{tabular}{|c|c|c|c|}
\hline 144 & 2 & 1 & 3 \\
\hline 1 & 137 & 10 & 2 \\
\hline 1 & 4 & 138 & 7 \\
\hline 0 & 0 & 4 & 146 \\
\hline
\end{tabular}

(d)
Figure 8. Confusion matrix for all the different configurations (a) Top arm configuration (b) Bottom arm configuration (c) Spaced ring configuration (d) Wrist ring configuration. All test values for all subjects are shown. The matrix shows the directions that are commonly confused. The darker values indicate where the stimuli was correctly identified with higher accuracy.

\section{\begin{tabular}{|l|l|l|l|}
\hline Up & Left & Down & Right \\
\hline
\end{tabular}}

\begin{tabular}{|c|c|c|c|c|}
\hline Up & 98.7 & 1.33 & 0 & 0 \\
\hline Left & 0 & 98.7 & 0.67 & 0.67 \\
\hline Down \\
\hline 0 & 0.67 & 98.7 & 0 \\
\hline Right \\
\hline 0 & 0 & 2.67 & 97.3 \\
\hline
\end{tabular}

Figure 9. Confusion matrix for the final chosen configuration (spaced-ring configuration) provided in percentage. The activated motor (stimuli) is listed along the vertical axis. The user response is listed along the horizontal axis. The matrix shows high accuracy and little confusion for different directions, with no significant bias in any of the 4 directions.

\section{B. User Response Speed}

The participants were asked to press the arrow keys as fast and accurately as they could. The user response time was defined as the time it took for the user to respond to the activated motor after an activation command was sent. The timestamp (computer time) when the motor activation command was sent (through BLE) was recorded along with the timestamp when the user pressed a key, and the difference was computed. This includes the time for BLE data transfer, motor response speed, and user's cognitive load in identifying the direction and then performing the action (pressing a key, in this case).

The participants used least amount of time to respond to the motor activations provided through spaced-ring configuration with $75 \%$ of the responses being within 1 second. The spacedring configuration also had the lowest variability in the user response time among the 4 configurations (Fig. 10).

To identify if participants were taking significantly longer to classify a direction, the variability among all directions for each of the configurations was analyzed (Fig. 11). The down 
direction took significantly more time in the top-arm configuration and the up direction in the bottom-arm configuration. It is interesting to observe that the most often misclassified direction also required a significantly larger time for the users to respond. The longer response times in the direction of most confusion suggests that the participant is aware of the vibrational sensation but is not able to clearly distinguish the direction and hence takes more time.

There was no significant difference in the response time among the 4 directions for the spaced-ring configuration, although the up and down directions required least time while the left and right directions showed a larger variability (Fig. 11).

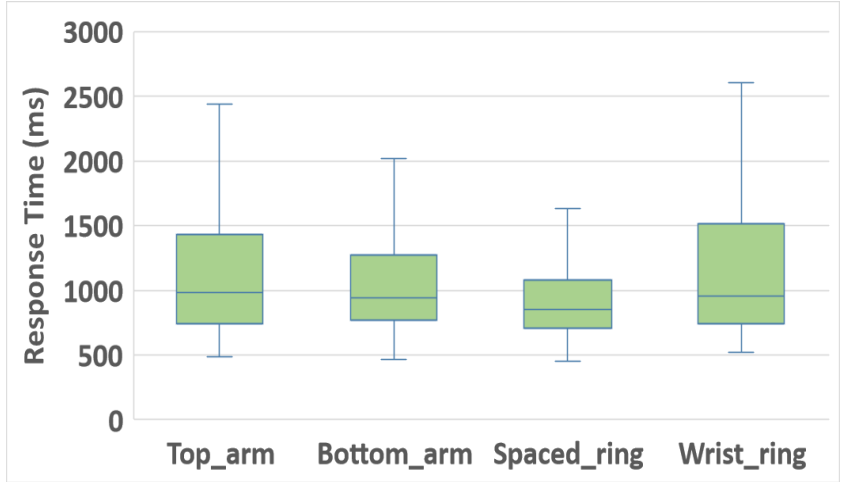

Figure 10. User response speed for different configurations.

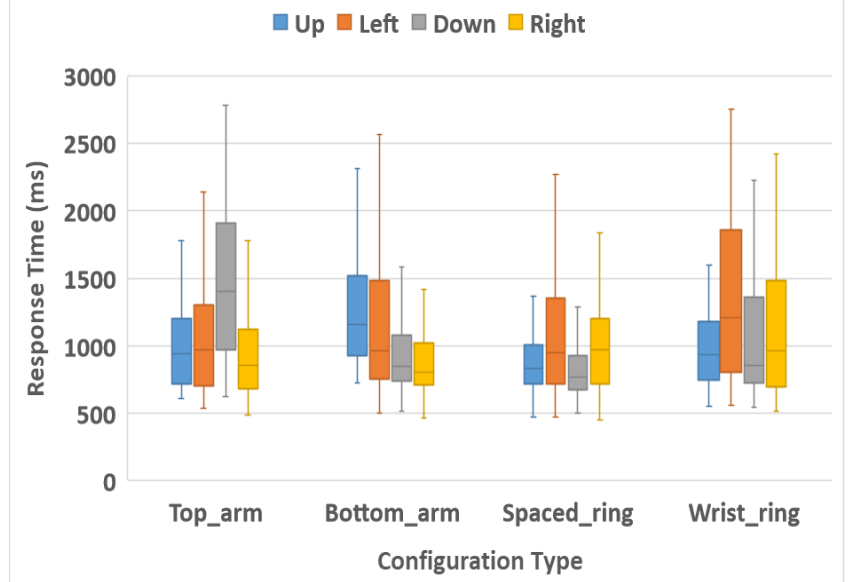

Figure 11. Directional variability for different configurations.

\section{A. Overall System}

Since the spaced-ring configuration produced the least confusion and had the lowest response time, it was chosen as the final design for the sleeve. The participants attempted to trace the letter displayed on the screen, by drawing on the glass using the marker. The participants were healthy adults and did not have any motor disabilities, hence this was a preliminary testing to study the proof-of-concept (Fig. 12).

It was observed that the system was able to track the user's hand movement and activate the correct motors corresponding to the deviation with a perceptible difference in the vibrational intensity as the hand moved away from (or closer to) the original trajectory (Fig. 13). The hand tracking was observed to occur in real-time and the feedback from the motors felt intuitive in directing the user towards the correct path.
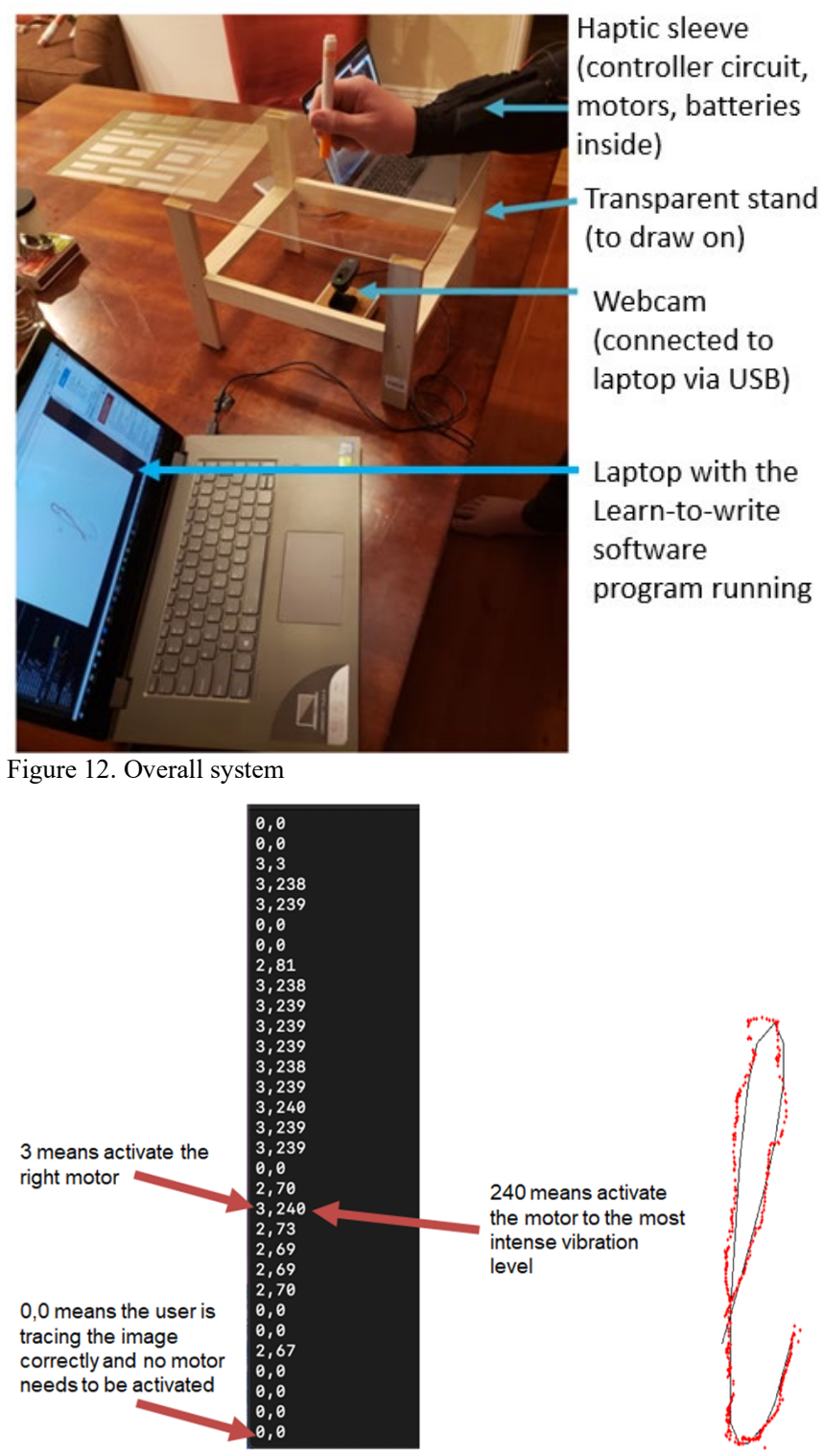

Figure 13 (a) Distance and deviation data sent to ESP32 as the hand moves (b) Representative displayed letter including the visual feedback provided to the participant.

\section{DISCUSSION}

\section{A. Sleeve Architecture}

In the post-testing feedback, all participants said that they found the spaced-ring configuration more intuitive and easier to distinguish directions. This ties to the lower user response speed measured in this configuration. The subject-to-subject difference in user response was also observed to be lowest in the spaced ring configuration. Given that there were only 3 participants in this study, there needs to be additional data to confirm this observation.

Although vibrotactile sleeves have not been used for handwriting training, much of the literature on using vibrating motor for hand guidance place the tactors around the 
wrist/forearm in a ring structure [20 -23]. Other studies present sleeves with several tactors $(16+)$ utilizing a grid structure with tactors equally spaced throughout the sleeve such that there is a row of tactors on the 4 quadrants [24, 25] or multiple rows on the dorsal and volar side of the forearm [26]. A ring structure is promising in designs with space concern, and the multitactor grid structure is advantageous for providing high data transfer. In cases where the user can wear a sleeve without hindering their activity, and the amount of information to be delivered through the vibrations is limited, the spaced-ring structure provides the benefit of the most accurate user interpretation and fastest response to the information provided.

Based on this study, we recommend the following considerations for tactor placement:

1. The up direction is best represented on the top (dorsal) side of the wrist/forearm.

2. The down direction is best represented on the bottom (volar) side of the wrist/forearm.

3. Tactors are better spread out on the arm, instead of a ring structure, for higher intuitiveness (better accuracy and speed).

\section{B. Wireless Communication Architecture}

In the initial design, the wireless communication between the learn-to-write software and the ESP32 was brokered through a web server Mosquitto. The distance and direction information were uploaded to the web server, and the microcontroller continuously monitored the server and retrieved this data. However, occasionally, some latency (1-2s) was observed between the marker deviating from the pattern and the haptic feedback, subject to the server connectivity. This latency issue was resolved by redesigning the system to use BLE for communication.

\section{CONCLUSION \& FUTURE WORK}

A low-cost wearable vibro-tactile system has been proposed for handwriting correction/training. The user wears a sleeve embedded with 4 vibrating motors, an ESP32 controller and a $3.3 \mathrm{~V}$ battery. The user attempts to trace a pattern/letter displayed on the computer screen, using a marker on a transparent glass. The marker location is captured by the webcam and displayed on the screen as a dot. Based on the deviation of the marker from the actual pattern, real-time data is sent to the ESP32 through BLE. The data is provided to the user in the form of the motor activation, with vibrational intensity increasing as they deviate further from the pattern.

Robotic therapy research has shown that haptic feedback based on error-augmentation (vibration increasing with increasing error / further from pattern) is more effective in poststroke training than feedback based on error-reduction (vibration increasing with reducing error / closer to pattern) [27]. Our system is currently based on error-augmentation but could be easily modified to also study the effectiveness of the feedback protocols in handwriting training.

The sleeve design has been characterized for different tactor configurations, and a novel arrangement for the tactor configurations based on the direction identification accuracy and response speed of the user has been presented. The 4 configurations considered are wrist-ring arrangement (commonly used), top-arm arrangement, bottom-arm arrangement, and spaced-ring arrangement. The spaced-ring configuration produced most accurate location discrimination with the fastest user response time. In the spaced-ring arrangement, a motor is embedded on each side of the forearm: up motor on the dorsal side near the wrist, down motor on the volar side near the proximal region $10 \mathrm{~mm}$ away from wrist, left motor on the lateral side $5 \mathrm{~mm}$ away from wrist, and right motor on medial side $5 \mathrm{~mm}$ away from wrist. To the best of the authors' knowledge, the spaced-ring configuration has not been presented previously.

The system needs to be tested on more subjects and efficacy of the system on users with learning disability or motor skills disability needs to be tested. Also, while the accuracy and speed response of sleeve have been characterized, the user ability to identify intensity level changes still needs to be characterized. The spaced-ring structure could be used for intensity and large-scale characterization in the future (once COVID restrictions on human subject testing are eased).

The system can be utilized to evaluate the handwriting patterns of individual users (based on the time taken to complete a pattern, number of missed pixels, average distance, and deviation from the pattern) and provide a score/report on their performance. This data may be used in tracking improvements over time with respect to control groups performing the same tasks using Phantom Omni based systems.

\section{REFERENCES}

[1] W. Park, et al., "Evaluation of Handwriting Skills in Children with Learning Difficulties.” In: Antona M., Stephanidis C. (eds) HumanComputer Interaction., HCII 2019, vol. 11573. pp 150-159.

[2] J. Mullins, C. Mawson and S. Nahavandi, "Haptic handwriting aid for training and rehabilitation," IEEE International Conference on Systems, Man and Cybernetics, 2005, vol. 3, pp. 2690-2694.

[3] F. Bara, \& E. Gentaz, "Haptics in teaching handwriting: The role of perceptual and visuo-motor skills", Human movement science, Aug. 2011, vol. 30. pp. 745-759.

[4] S. Chang, S. and N. Yu, "Visual and haptic perception training to improve handwriting skills in children with dysgraphia.", The American Journal of Occupational Therapy, 2017, vol. 17(2).

[5] S. Fisackerly et al., "Haptic-enabled virtual handwriting: Multimodal handwriting instructions for young students," IEEE Symposium on Haptic Audio-Visual Environments \& Games, 2010, pp. 1-6.

[6] C. L. Teo, E. Burdet and H. P. Lim, "A robotic teacher of Chinese handwriting," Symposium on Haptic Interfaces for Virtual Environment and Teleoperator Systems, 2002, pp. 335-341.

[7] Y. Kim et al., "Haptics Assisted Training (HAT) System for children's handwriting," World Haptics Conference (WHC), 2013, pp. 559-564.

[8] A. Teranishi, G. Korres, W. Park and M. Eid, "Combining Full and Partial Haptic Guidance Improves Handwriting Skills Development," IEEE Transactions on Haptics, 2018, vol. 11, no. 4, pp. 509-517.

[9] W. Park, G. Korres, T. Moonesinghe and M. Eid, "Investigating Haptic Guidance Methods for Teaching Children Handwriting Skills," IEEE Transactions on Haptics, 2019, vol. 12, no. 4, pp. 461-469.

[10] L. Jing, Z. Dai and Y. Zhou, "Wearable Handwriting Recognition with an Inertial Sensor on a Finger Nail," IAPR International Conference on Document Analysis and Recognition, 2017, pp. 1330-1337.

[11] X. Lin, et al., "SHOW: Smart handwriting on watches", Interactive, Mobile, Wearable and Ubiquitous Technologies, Jan. 2018, pp 1-23.

[12] C. Amma, et al. "Airwriting: A wearable handwriting recognition system", Personal and Ubiquitous Computing, Jan. 2014, vol. 18.

[13] Available at: https://www.especialneeds.com

[14] Available at: https://therapyshoppe.com/category/P1822-weightedhandwriting-glove-writing-tools-sensory-products-tools-fidget 
[15] A. Morikawa, et al., "Double Pressure Presentation for Calligraphy Self-training", Companion of the 2018 ACM/IEEE International Conference on Human-Robot Interaction, Mar. 2018, pp. 199-200.

[16] M. Narita, and T. Matsumaru, "Calligraphy-stroke learning support system using projection", IEEE International Workshop on Robot and Human Interactive Communication, Nov. 2015, pp 640-645.

[17] M. Matscheko, A. Ferscha, A. Riener, M. Lehner, “ Tactor placement in wrist worn werables." International Symposium on Wearable Computers (ISWC), Oct. 2010.

[18] R. W. Cholewiak and A. A. Collins, "Vibrotactile localization on the arm: Effects of place, space, and age," Perception \& psychophysics, 2003 , vol. 65 , no. 7 , pp. 1058-1077.

[19] S. Weinstein, "Intensive and extensive aspects of tactile sensitivity as a function of body part, sex, and laterality," The skin senses, K. DR., Ed., Springfield, 1968, pp. 195-218.

[20] F. Sergi, D. Accoto, D. Campolo and E. Guglielmelli, "Forearm orientation guidance with a vibrotactile feedback bracelet: On the directionality of tactile motor communication," 2nd IEEE RAS \& EMBS International Conference on Biomedical Robotics and Biomechatronics, Scottsdale, AZ, USA, 2008.

[21] J. Salazar, K. Okabe and Y. Hirata, "Path-Following Guidance Using Phantom Sensation Based Vibrotactile Cues Around the Wrist," IEEE Robotics and Automation Letters, July 2018, vol. 3, no. 3, pp. 24852492.

[22] J. Hong, A. Pradhan, J. E. Froehlich, and L.. Findlater, "Evaluating Wrist-Based Haptic Feedback for Non-Visual Target Finding and Path Tracing on a 2D Surface." Proceedings of the 19th International ACM SIGACCESS Conference on Computers and Accessibility (ASSETS), 2017, New York, USA.

[23] K. Bark et al., "Effects of Vibrotactile Feedback on Human Learning of Arm Motions," IEEE Transactions on Neural Systems and Rehabilitation Engineering, Jan. 2015, vol. 23, no. 1, pp. 51-63.

[24] D. Prabhu, M. M. Hasan, L. Wise, C. MacMahon and C. McCarthy, "VibroSleeve: A wearable vibro-tactile feedback device for arm guidance," 42nd Annual International Conference of the IEEE Engineering in Medicine \& Biology Society (EMBC), Montreal, Canada, 2020, pp. 4909-4912.

[25] T. McDaniel, D. Villanueva, S. Krishna and S. Panchanathan, "MOVeMENT: A framework for systematically mapping vibrotactile stimulations to fundamental body movements," IEEE International Symposium on Haptic Audio Visual Environments and Games, Phoenix, USA, 2010, pp. 1-6.

[26] J. S. Martinez, L. L. Holt, C. M. Reed and H. Z. Tan, "Incidental Categorization of Vibrotactile Stimuli," IEEE Transactions on Haptics, vol. 13, no. 1, pp. 73-79, 1 Jan.-March 2020.

[27] L. Y. Liu, et al., "The effects of error-augmentation versus errorreduction paradigms in robotic therapy to enhance upper extremity performance and recovery post-stroke: a systematic review. " Journal of neuroengineering and rehabilitation, Jul. 2018, vol. 15. 